\title{
Estresse ocupacional em profissionais de enfermagem
}

\author{
Occupational stress in nursing personnel \\ Estrés ocupacional en profesionales de enfermería
}

\author{
Eliana Ofélia Llapa-Rodriguez'; Júlian Katrin Albuquerque de Oliveira"; David Lopes Neto"I; \\ Cristiane Franca Lisboa Gois'v; Maria Pontes de Aguiar Camposv; Maria Claúdia Tavares de Mattos ${ }^{v 1}$
}

\begin{abstract}
RESUMO
Objetivo: analisar os fatores de estresse em enfermeiros de um hospital universitário da cidade de Aracaju, Sergipe, Brasil. Método: pesquisa descritiva e correlacional. A amostra foi composta por 101 profissionais de enfermagem. Os dados foram coletados pela técnica de entrevista, com aplicação do Inventário para Estresse em Enfermeiros e posteriormente analisados utilizando o teste $t$ de Student para amostras independentes. O projeto do estudo foi aprovado por Comitê de Ética em Pesquisa. Resultados: $43 \%$ dos itens analisados foram fontes de estresse ocupacional entre os profissionais de enfermagem, sendo o fator papéis estressores da carreira o responsável por maior influência ao estresse (54\%). O local de trabalho foi a única variável que apresentou diferença significativa quando associada aos fatores analisados. Conclusão: a enfermagem se revelou como uma profissão de risco para o estresse ocupacional, sofrendo diversas influências dos fatores analisados, relacionados à organização e ao processo de trabalho.
\end{abstract}

Descritores: Esgotamento profissional; equipe de enfermagem; estresse psicológico; saúde do trabalhador.

\section{ABSTRACT}

Objective: : to examine stress factors in nurses at a university hospital in Aracaju, Sergipe, Brazil. Method: in this descriptive, correlational study, data were collected by interview from a sample of 101 nursing personnel, by applying a Nursing Stress Inventory, and analyzed using Student's t-test for independent samples. The study was approved by the research ethics committee. Results: $43 \%$ of the items examined were sources of occupational stress among nursing professionals, with stressful career roles being responsible for most influence on stress (54\%). Work place was the only variable that displayed significant difference when associated with the factors examined. Conclusion: Nursing revealed itself to be a profession with risk of occupational stress, which was influenced in various manners by the work organization and work process factors examined.

Descriptors: Burnout, professional; nursing team; stress, psychological; occupational health.

\section{RESUMEN}

Objetivo: analizar los factores de estrés en enfermeros de un hospital universitario de la ciudad de Aracaju, Sergipe, Brasil. Método: investigación descriptiva y correlacional. La muestra se compuso de 101 profesionales de enfermería. Los datos fueron recolectados por la técnica de entrevista, con aplicación del Inventario para Estrés en Enfermeros y, posteriormente, analizados utilizando la prueba t de Student para muestras independientes. El proyecto de estudio fue aprobado por el Comité de Ética en Investigación. Resultados: El 43\% de los ítems analizados fue fuente de estrés ocupacional entre los profesionales de enfermería, siendo el factor papeles estresores de la carrera el responsable por mayor influencia al estrés (54\%). El lugar de trabajo fue la única variable que presentó diferencia significativa cuando se asoció a los factores analizados. Conclusión: la enfermería se reveló como una profesión de riesgo en cuanto al estrés ocupacional, sufriendo diversas influencias de los factores analizados, relacionados a la organización y al proceso de trabajo. Descriptores: Agotamiento profesional; grupo de enfermería; estrés psicológico; salud laboral.

\section{INTRODUÇÃO}

O estresse é considerado o mal do século 21 pela Organização Mundial de Saúde (OMS). Nesse contexto, o estresse vem sendo considerado como uma doença relacionada ao trabalho em âmbito mundial. Pela magnitude do problema, tem se tornado alvo de pesquisas das áreas sociais e da saúde ao ser causador de efeitos psicológicos e fisiológicos nocivos ao trabalhador ${ }^{1,2}$.
O local de trabalho é um ambiente que faz parte da vida das pessoas e, inevitavelmente, causa estresse. O ser humano para promover sua adaptação frente a estimulos hostis desencadeia alterações neuroendócrinas que possibilitam sua reorganização com a finalidade de manter sua homeostase ${ }^{3}$. Considerado como resposta a demandas externas decorrentes de pressões que

\footnotetext{
'Enfermeira. Doutora. Professora Associada, Universidade Federal de Sergipe. Brasil. E-mail: elianaofelia@gmail.com. "Enfermeira. Mestre. Faculdade Mauricio de Nassau. Aracaju, Sergipe, Brasil. E-mail: jukatrin@yahoo.com.br.

"'Enfermeiro. Doutor. Professor Associado,Universidade Federal do Amazonas. Escola de Enfermagem de Manaus. Brasil. E-mail: davidnetto@uol.com.br. IVEnfermeira. Doutora. Professora Adjunta, Universidade Federal de Sergipe, Brasil. E-mail: cristianeflg@hotmail.com.

vEnfermeira. Doutora. Professora Associada, Universidade Federal de Sergipe, Brasil. E-mail: mapacampos@gmail.com.

v'Enfermeira. Doutora. Professora Associada, Universidade Federal de Sergipe. Brasil. E-mail: mctm@ufs.br.
} 
excedem a capacidade das pessoas de resistir e manter seu estado natural, esta forma de esgotamento tem ocasionado problemas fisiológicos e gerenciais, entre eles doenças do trabalho, turnover e absenteísmo².

Tendo em vista que as doenças relacionadas ao trabalho comprometem a saúde do trabalhador, busca-se então compreender as causas que propiciam estresse. Desta forma, levantou-se a seguinte questão de pesquisa: quais os fatores têm predisposto os profissionais de enfermagem a esta condição?

Destarte, o estudo poderá servir de subsídios para construção de políticas públicas voltadas para a saúde do trabalhador e notadamente dos profissionais de enfermagem. Sendo assim, o objetivo desta pesquisa foi analisar os fatores de estresse em enfermeiros de um hospital universitário do município de Aracaju, Sergipe, Brasil

\section{REVISÃo DE LITERATURA}

Estresse ocupacional é definido como um estado reacional biológico deletério e caracterizado como um agravamento multifatorial ${ }^{1}$ decorrente de processos interacionais entre o trabalhador e seu ambiente ${ }^{4}$. Este estado tem origem a partir de múltiplos fatores de risco pessoais ${ }^{5}$, ambientais, biológicos, psicológicos, sociais e organizacionais que prejudicam à qualidade de vida dos trabalhadores da saúde, afetando, principalmente, profissionais de enfermagem ${ }^{6}$.

Pesquisadores explicam que individuos ao assumirem muitos papéis na vida usualmente enfrentam grande peso, tanto na vida familiar como no trabalho, gerando conflitos em relação ao seu ambiente laboral ${ }^{7}$. Condições, principalmente, em decorrência, do exercício laboral com longas jornadas de trabalho e prestação de cuidados a pessoas em situação de vulnerabilidade (biológica e psicológica, com sofrimentos psíquicos e estados terminais) ${ }^{6}$.

Historicamente, estudos sobre o estresse ocupacional têm mostrado sequencialmente um aumento na sua prevalência, despertando, mundialmente, o interesse de cientístas em avaliá-lo como problemática de saúde pública ${ }^{8}$, especialmente, quanto a mensuração de fatores determinantes, eventos e estímulos estressores no processo de trabalho $0^{3,7,9,10}$

Devido ao estatuto laboral sui generis, com o passar dos anos, o serviço desempenhado pela enfermagem desencadeia sinais e sintomas de doenças ocupacionais. Estes afetam enfermeiros, técnicos e auxiliares de enfermagem alterando sua capacidade de trabalho e propiciando o surgimento de exaustão emocional, irritabilidade, síndrome da fadiga crônica, distúrbios do sono, transtornos depressivos, síndrome de esgotamento profissional e estresse ocupacional ${ }^{11}$.

Portanto, o estresse no trabalho do profissional de enfermagem é um fenômeno constante e disseminado nos diferentes postos de atuação de enfermeiros, técnicos e auxiliares de enfermagem, estando diretamente relacionado ao ambiente e às condições de trabalho alterando, assim, seu estado de saúde física e mental ${ }^{7}$.

\section{Metodologia}

Estudo com enfoque quantitativo, com delineamento descritivo e correlacional, desenvolvido no hospital universitário do Estado de Sergipe, Brasil. A população do estudo foi constituída por 123 profissionais de enfermagem e como critério de inclusão foi exigido no mínimo seis meses na assistência direta. A amostra, intencional e não probabilística, foi constituída de 101 profissionais de enfermagem, sendo 21 enfermeiros e 80 técnicos/auxiliares de enfermagem, lotados em quatro clínicas (médica I e II, cirúrgica e pediátrica). O restante de profissionais, parte da população, não foram incluidos, devido a que não se enquadravam nos critérios estabelecidos.

Como instrumento de coleta foi utilizado o questionário denominado Inventário de Estresse em Enfermeiros (IEE) ${ }^{12}$, composto de 44 itens, com cinco opções de respostas, desde nunca (1) até sempre (5), detalhados a seguir: 1으 fator - relações interpessoais (com 17 itens); 2 음 fator - papéis estressores da carreira (com 11 itens); 3 은 fator - intrínseco ao trabalho (com 10 itens). Somam-se a esses fatores mais seis itens isolados, os quais foram discriminados dos demais por não apresentarem comunalidade, ressalta-se que estes últimos são considerados pela literatura como imprescindíveis para a determinação do estresse. O questionário IEE foi autoaplicado e apresentou duas partes, a primeira, relativa à caracterização dos profissionais e a segunda, contendo os três fatores, acima descritos, e o grupo de itens isolados.

A coleta de dados foi realizada nos meses de setembro de 2013 a fevereiro de 2014. Os profissionais foram abordados individualmente em seus locais de trabalho e esclarecidos quanto aos aspectos metodológicos e éticos da pesquisa, apresentando os objetivos, o anonimato, os riscos e os benefícios. Após a assinatura do Termo de Consentimento Livre e Esclarecido, foram entregues aos participantes os instrumentos de coleta de dados, e posterior ao prazo de sete dias foram devolvidos para análise. O protocolo teve aprovação pelo Comitê de Ética em Pesquisa da Universidade Federal de Sergipe, CAAE: 20668713.4.00005546.

Os dados foram analisados por meio do teste $t$ de Student, para amostras independentes, sendo o nível de confiança de $95 \%$ e o de significância de $5 \%$, com valor de $p<0,05$ para indicar a existência de diferença estatisticamente significativa.

\section{RESUltados e Discussão}

O estresse ocupacional emerge no campo da saúde como um problema real entre os profissionais de enfermagem, devido a característica exaustiva do trabalho e as diversas atribuições desenvolvidas por 
estes profissionais. Isto posto, a enfermagem vivencia, diariamente, situações de exaustão física e emocional somada à administração excessiva e geradora de conflitos organizacionais, que expõe enfermeiros a fatores determinantes de estresse ocupacional ${ }^{13-15}$.

Quanto aos profissionais participantes deste estudo, constatou-se que a maioria eram do sexo feminino $90(89,1 \%)$, pertencentes à categoria de técnico/auxiliar de enfermagem $80(79,2 \%)$, seguidos da categoria de enfermeiros $21(20,8 \%)$. Com relação ao local de trabalho, $31(30,7 \%)$ trabalhavam na clínica cirúrgica, $27(26,7 \%)$ na clínica médica I, $26(25,7 \%)$ na clínica médica II e $17(16,8 \%)$ na clínica pediátrica. Quanto ao turno de trabalho, o período noturno foi o que apresentou maior número de profissionais $44(43,6 \%)$, seguido dos turnos matutino - 31(30,7\%) e vespertino - $26(25,7 \%)$.

Os resultados obtidos permitiram identificar todos os fatores como predisponentes para o estresse ocupacional. No entanto, o mais preponderante foi o fator papéis estressores da carreira, o qual apresentou $54 \%$ de itens como significativos, seguido do grupo de itens isolados (50\%), intrínseco ao trabalho (40\%) e relações interpessoais (35\%).

Estudo mostra que fragilidades no relacionamento com a equipe de trabalho pode gerar situações de estresse tornado o profissional emocionalmente fragil, débil e sensível, fato ocasionado por fatores extrinsecos ao relacionamento tais como desgaste e sobrecarga no ambiente de trabalho e falta de recursos humanos e materiais. Ainda, destacam-se fatores subjetivos, tais como inefetivo relacionamento com o paciente e seus familiares, falta de comunicação, relacionamento negativo com a chefia, desunião da categoria e dificuldade no gerenciamento de pessoas ${ }^{16}$.

Na análise de cada um dos fatores com as variáveis em estudo, foi possível identificar associação significativa entre o fator papéis estressores da carreira com a variável turno (matutino e noturno) no item $30(0,049)$ - distanciamento entre a teoria e a prática. Ainda no fator papéis estressores da carreira, a variável local de trabalho foi significativa, nas clínicas médica I e II, no item 37(0,01) sentir-se impotente diante das tarefas a serem realizadas; entre as clínicas médica I e cirúrgica nos itens 30(0,034) e $39(0,001)$ - indefinição do papel do enfermeiro; entre as clínicas médica I e pediátrica nos itens $34(0,032)$ - ter um prazo curto para cumprir ordens, $35(0,036)$ - restrição da autonomia profissional, 37(0,024) e 39(0,000); entre as clínicas médica II e cirúrgica no item 39(0,034), e entre as clínicas médica II e pediátrica nos itens 39(0,021) e $41(0,048)$ - impossibilidade de prestar assistência direta ao paciente, por serem significativos.

A respeito do trabalho noturno, estudo realizado em unidade de terapia intensiva, identificou insatisfação dos profissionais devido a necessidade de ter atenção e alerta redobrada no cuidado do paciente e sobrecarga relacionada à falta de profissionais que trabalham no turno matutino, situações que provocam no profissional maior desgaste fisico e cognitivo ${ }^{17}$, entendidos como situações que podem levar a estresse profissional.

Quanto ao prazo curto para cumprir ordens relacionadas ao trabalho, estudo sobre estresse e distúrbios psíquicos, em trabalhadores de enfermagem, mostrou que o tempo e a velocidade para realização das ações de enfermagem geram pressões psicológicas, e, portanto predispõem ao estresse ocupacional ${ }^{18}$. O dispêndio mínimo de tempo para execução de ações de enfermagem foi identificado, em estudo realizado com 143 enfermeiros de um hospital universitário, como maior fator de estresse no gerenciamento hospitalar, corroborando com os achados deste estudo ${ }^{19}$.

A escassez de tempo para a realização das atividades assistenciais e alguns fatores como o elevado número de pacientes, redução do quantitativo de recursos humanos e sobrecarga de trabalho podem levar a prazos curtos para o cumprimento de ações, comprometendo a qualidade da assistência, e levando à exaustão física e emocional.

Por outro lado, estudo com 3.471 trabalhadores de enfermagem, de cinco hospitais públicos universitários de diferentes regiões do país, mostrou que a indefinição de papéis ocasionada pela falta de autonomia do enfermeiro na resolutividade de ações gerenciais e assistenciais, influencia, sobremaneira, no estresse, independente da exposição a diferentes cargas psíquicas ${ }^{20}$.

$\mathrm{Na}$ análise do grupo itens isolados, observou-se significância apenas com a variável local de trabalho, quando relacionada às clínicas médicas I e II no item $44(0,044)$ - receber este salário; entre as clínicas médica I e pediátrica no item $1(0,046)$ - começar em uma função nova, nas clínicas médica II e cirúrgica no item 19(0,042) - fazer turnos alternados de trabalho e nas clínicas médica II e pediátrica com o item $1(0,041)$.

Em sintonia com esses resultados, estudo sobre tensões ocupacionais na enfermagem, revelou que o baixo salário foi considerado como fonte de estresse, devido principalmente ao cumprimento de dupla jornada de trabalho ${ }^{21}$. Estudos mostram que a sobrecarga, o ritmo de trabalho, as dobras de plantão e a existência de salários injustos podem afetar o desempenho físico, mental e emocional, e resultam em exaustão e despersonalização 22,23 na vida dos profissionais de enfermagem. Por outro lado, supõe-se que estando o profissional satisfeito com seu ambiente de trabalho, nas relações com os colegas, entre outras variáveis de satisfação, há probabilidade de que estas situações positivas transformem as pressões em fonte de crescimento emocional e profissional.

Com relação ao fator intrínseco ao trabalho, observou-se significância com a variável sexo, com destaque para o item $6(0,005)$ - fazer esforço físico para cumprir o trabalho. Quanto ao local de trabalho, houve significância entre as clínicas médica I e pediátrica, nos itens $10(0,027)$ - levar serviço para fazer em casa, e 15(0,029) - falta de recursos humanos; entre as clínicas médica II e 
cirúrgica os itens 6(0,042) e 9(0,007) - cumprir na prática uma carga horária maior; entre as clínicas médica II e pediátrica os itens $6(0,028), 9(0,014)$ e $15(0,037)$; e entre as clinicas cirúrgica e pediátrica, o item $10(0,039)$.

Em conformidade com estes achados, estudo que aplicou o mesmo instrumento junto a profissionais da saúde, no Estado da Paraíba, identificou como fonte de estresse, a exaustão física durante o desenvolvimento de atividades terapêuticas de diferente natureza, situação que interferia significativamente na qualidade da assistência e na produtividade desses profissionais ${ }^{24}$. Por outro lado, investigação em uma UTI neonatal demonstrou que o número reduzido de funcionários foi fator de estresse entre os profissionais, interferindo no ritmo de trabalho e na qualidade da assistência ${ }^{25}$. Ainda, tem sido identificado por meio de pesquisa que profissionais de enfermagem desenvolvem altos índices de fatiga emocional, estresse e burnout quando as atividades envolviam cuidados críticos, paliativos e dilemas éticos ${ }^{9,10,26}$. A respeito, os recursos humanos desempenham papel fundamental nos serviços de saúde e sua inadequada distribuição limitam sobremaneira o desempenho do profissional justificando sua significância e influência nos fatores de estresse analisados.

Dentro da mesma lógica, estudo irlandês, mostrou que assegurar um quantitativo adequado de profissionais, levando-se em consideração as necessidades do paciente, contribuiu para a redução do estresse ocupacional ${ }^{27}$ Este achado, mais uma vez reafirma a necessidade da adequação dos recursos humanos com o intuito de evitar que o profissional de enfermagem tenha uma carga de trabalho muito desgastante. As análises mostraram que quando associado o fator relações interpessoais com a variável turno (matutino e noturno), os itens $14(0,040)$ - manter-se atualizada e $25(0,007)$ - relacionamento com a chefia, foram significativos. Já na associação com a variável local de trabalho, relacionado à clínica médica I e II, ressaltou-se o item 33(0,041) - executar procedimentos rápidos; e para as clínicas médica I e cirúrgica, os itens $33(0,016)$ e 40(0,047) - responsabilizar-se pela qualidade de serviço que a instituição presta. Para as clínicas médica I e pediátrica, destacaram-se os itens $42(0,024)$ - a especialidade em que trabalho e $43(0,006)$ - atender um número grande de pessoas; para as clínicas médica II e cirúrgica salientando-se o item $14(0,035)$ e, por fim, distinguiu-se o item 42(0,049) entre as clínicas pediátrica e cirúrgica, por serem relevantes para este estudo.

Estes resultados assemelharam-se a outros estudos, já que mostram o excessivo número de pacientes como uma das principais causas para o estresse ocupacional. Ainda, nesses artigos, foi observado, que o processo de trabalho, as cargas psíquicas e os desgastes gerados em trabalhadores de enfermagem estão relacionados ao número insuficiente de profissionais e às fragilidades no gerenciamento ${ }^{20,25,28}$. Fatores que levam a um ritmo acelerado de trabalho, e, por conseguinte, a um menor tempo para repouso e organização das atividades assistenciais ${ }^{20}$

Por outro lado, o exercício do gerenciamento requer o desenvolvimento de habilidades e de competências do tipo interpessoal, sendo apontado o relacionamento com a equipe, como uma das maiores dificuldades no desempenho de atividades gerenciais. Esta situação pode configurar-se como desencadeante de exaustão, de desgaste físico e de insatisfação no trabalho ${ }^{29,30}$. A partir dessa reflexão, destaca-se a necessidade de o enfermeiro, como gerente e líder nato da equipe de enfermagem, desenvolver estratégias que favoreçam a negociação e a resolução de conflitos, de maneira tal que a equipe possa trabalhar integrada e em sintonia com os objetivos profissionais e organizacionais para redução do estresse ocupacional.

\section{CONCLUSÃo}

A enfermagem revelou-se como uma profissão de risco para o estresse ocupacional, sofrendo diversas influências relacionadas à organização e ao processo de trabalho. $O$ fator papéis estressores da carreira foi o responsável pela maior influência geradora do problema, seguido do grupo de itens isolados, do fator intrínseco ao trabalho e do fator relações interpessoais.

A variável local de trabalho se destacou por ser a única que influenciou diretamente em todos os fatores estudados. A variável turno apresentou relação com o estresse quando associada aos fatores relações interpessoais e papéis estressores da carreira. Os turnos matutino e noturno indicaram as maiores influências para o quesito em estudo. A variável sexo foi significativa quando associada ao fator intrínseco ao trabalho. A inadequada distribuição de recursos humanos foi fator determinante para a maioria das respostas entre os fatores estudados.

Frente aos resultados, é preciso criar estratégias gerenciais que possibilitem mitigar os efeitos estressantes causados pelos fatores analisados, tais como: adequação do quantitativo profissional, implementação de melhorias nas ações de gerenciamento, distribuição adequada da escala de serviço, comunicação efetiva entre as equipes, melhoria das condições de trabalho e educação continuada.

A principal limitação do estudo foi a tipologia transversal, o que permitiu deter-se na análise de frequências e associações dos itens constituintes do instrumento utilizado, sem a possibilidade de estabelecer relações causais. Recomenda-se que sejam realizadas pesquisas quantitativas mais abrangentes e qualitativas com profundidade temática, nos diferentes níveis de assistência, com a finalidade de ampliar o conhecimento sobre o estresse ocupacional na enfermagem. 


\section{REFERÊNCIAS}

1. Teixeira CAB, Pereira SS, Cardoso L, Seleghim MR, Reis LN, Gherardi-Donato ECSG. Occupational stress among nursing technicians and assistants: coping focused on the problem. Invest.Educ. Enferm. [Internet]. 2015 [cited 2017 Oct 18]; 33:28-34.Available from: http://www.scielo.org.co/pdf/iee/v33n1/v33n1a04.pdf. 2. Jayashree R. Stress: management with special reference to public sector bank employees in chennal. IJEIMS. [Internet]. 2010 [cited 2017 Mar 06]; 1(3):34-9. Available from: http://www.ijcns. com/pdf/34-39.pdf.

3. Trettene AS, Ferreira JAF, Mutro MEG, Tabaquim MLM, Razera APR et al. Estresse em profissionais de enfermagem atuantes em Unidades de Pronto Atendimento. Bol. Acad. Paul. Psicol. [Internet]. 2016 [citado em 21 set 2018]; 36(91):243-61. Disponível em http:// pepsic.bvsalud.org/pdf/bapp/v36n91/v36n91a02.pdf.

4. Kim K, Lee S, Choi YH. Relationship between occupational stress and depressive mood among interns and residents in a tertiary hospital, Seoul, Korea. Clin. Exp. Emerg. Med. [Internet]. 2015 [cited 2017 Oct 13];2:117-22. Available from: http://ceemjournal. org/upload/pdf/ceem-15-002.pdf.

5. Latifzadeh S, Zarea K. Occupational stress and its related factors in nurses working in intensive care units of educational hospitals in Ahwaz, Iran. J. Nov. Appl. Sci. [Internet]. 2015 [cited 2017 Oct 13]; 4:483-7. Available from: https://www.researchgate.net/publication/277138938_Occupational_Stress_and_Its_Related_Factors_in_Nurses_Working_in_Intensive_Care_Units_of_Educational_Hospitals_in_Ahwaz_Iran

6. Avila IYC, Gomez AA, Tapias KC, Castro SL. Estrés laboral en enfermería y factores asociados Cartagena (Colombia). Salud Uninorte [Internet]. 2014 [citado 13 Oct 2017]; 30(1):34-43. Disponible en: http://www.redalyc.org/html/817/81730850005/

7. Fauzan A, Afnan TE, Achmad S, Mintarti R. The effect of role ambiguity towards the performance of nurses through organizational commitment: a study on regional general hospitalof dr. saiful anwar, malang, indonesia. RJOAS [Internet]. 2017 [cited 2017 Sep 22]; 6(66); 234-8. Available from: https://rjoas.com/issue-2017-06/ article 27.pdf

8. Yaacob M, Long CS. Role of Occupational stress on job satisfaction. Mediterr.J.Soc.Sci. [Internet]. 2015 [cited 2017 Oct 13]; 6(2):81-7. Available from: http://www.mcser.org/journal/index. $\mathrm{php} / \mathrm{mjss} /$ article/view/5867/5652

9. Al-Majid S, Carlson N, Kiyohara M, Faith M, Rakovski C. Assessing the degree of compassion satisfaction and compassion fatigue Among. J. Nurs. [Internet]. 2018 [cited 2018 Jan 21]; 48(6):310-15. Available from: https://insights.ovid.com/ pubmed?pmid $=29794595$

10. Caccamo F, Ghedin S, Marogna C. Evaluation of burnout and alexithymia in a group of nurses in palliative care: a pilot study. G. Ital. Med. Lav. Ergon. [Internet]. 2017 [cited 2017 Sep 21]; 39(4):249-55. Available from: https://www.ncbi.nlm.nih.gov/pubmed/29916571 11. Silva JL, Soares RS, Costa FS, Ramos DS, Lima FB, Teixeira LR. Fatores psicossociais e prevalência da síndrome de burnout entre trabalhadores de enfermagem intensivistas. Rev. bras. ter. intensiva. [Internet]. 2015 [citado em 15 fev 2017]; 27:125-33. Disponível em: http://www.scielo.br/pdf/rbti/v27n2/en_0103-507X-rbti-27-02-0125.pdf

12. Stacciarini JMR, Trócolli BT. Instrumento para mensurar o estresse ocupacional: Inventário de Estresse em Enfermeiros (IEE). Rev. latinoam. enferm. (Online). [Internet] 2000 [citado em 18 out 2017]; 8(6):40-9. Disponível em:http://www.scielo.br/pdf/rlae/ v8n6/12347.pdf

13. Fonseca JRF, Lopes Neto D. Níveis de estresse ocupacional e atividades estressoras em enfermeiros de unidades de emergência. Rev. Rene. [Internet]. 2014 [citado em 18 out 2017]; 15(5):732-42. Disponível em: http://periodicos.ufc.br/rene/article/view/3230/2486 14. Khamisa N, Oldenburg B, Peltzer K, Ilic D. Work related stress, burnout, job satisfaction and general health of nurses. Int. J. Environ Res. Public Health [Internet]. 2015 [cited 2017 Oct 13]; 12(1):652-66. Available from: https://www.ncbi.nlm.nih.gov/ pubmed/25588157

15. Dalmolin GL, Lunardi VL, Barlem ELD, Silveira RS. Implicações do sofrimento moral para os (as) enfermeiros (as) e aproximações com o burnout. Texto \& contexto enferm (Online) 2012 [citado em 04 out 2017]; 21:200-8. Disponível em: http://www.scielo.br/pdf/ tce/v21n1/en a 23v21n1.pdf
16. Araujo MPS, Medeiros SM, Quental LLC. Relacionamento interpessoal da equipe de enfermagem: fragilidades e fortalezas. Rev. enferm. UERJ [Internet]. 2016 [citado em 22 set 2018]; 24(5):7657. Disponível em:http://www.facenf.uerj.br/v24n5/v24n5a09.pdf 17. Silveira M, Camponogara S, Beck CLC, Costa VZ, Dalmolin GM, Arboit EL. Trabalho noturno e seus significados para enfermagem de unidade de cuidados intensivos. Rev. enferm. UERJ [Internet]. 2016 [citado em 22 set 2017]; 24(6):7416. Disponível em:http:// www.facenf.uerj.br/v24n6/v24n6a14.pdf

18. Urbanetto JS, Magalhaes MCC, Maciel VO, Sant'Anna VM, Gustavo AS, Poli-de-Figueiredo CE et al. Estresse no trabalho segundo o modelo demanda-controle e distúrbios psíquicos menores em trabalhadores de enfermagem. Rev. Esc. Enferm. USP. [Internet]. 2013 [citado em 28 set 2017]; 47(3):1186-93.Disponível em: http://www.scielo.br/pdf/reeusp/v47n5/pt_0080-6234 reeusp-47-05-1180.pdf

19. Guido LA, Linch GFC, Pitthan LO, Umann J. Estresse, coping e estado de saúde entre enfermeiros hospitalares. Rev. Esc. Enferm. USP [Internet]. 2011 [citado em 05 fev 2017]; 45(6):1427-31. Disponível em: http://www.scielo.br/scielo.php?script=sci arttext\&pid=S0080- 62342011000600022\&lng=en

20. Mininel VA, Baptista PCP, Felli VEA. Psychic workloads and strain processes in nursing workers of brazilian university hospitals. Psychic workloads and strain processes in nursing workers of brazilian university hospitals. Rev. latinoam. enferm. (Online) [Internet]. 2011 [cited 2017 Feb 15]; 19(2):340-7. Available from: http://www.scielo. $\mathrm{br} / \mathrm{pdf} / \mathrm{rlae} / \mathrm{v} 19 \mathrm{n} 2 / 16 . \mathrm{pdf}$

21. Cardoso MR, Lima SA, Oliveira DS, Viana PT, Merces MC, Andrade LM. Fatores estressores: interferência na qualidade da assistência dos profissionais enfermeiros. Prat. Hosp. [Internet]. 2015 [citado em 05 fev 2017]; 17:22-7. Disponível em: http://www.fufs.edu.br/ admin/anexos/12-08-2015_12_05_28_.pdf

22. Wang S, Liu Y, Wang L. Nurse burnout: personal and environmental factors as predictors. Int J. Nurs. Pract. [Internet]. 2015 [cited 2017 Oct 13]; 21(1):78-86. Available from: https://www.ncbi.nlm. nih.gov/pubmed/24237882

23. Cruz SP, Abellán MV. Desgaste profissional, stress e satisfação no trabalho do pessoal de enfermagem em um hospital universitário. Rev. latinoam. enferm. (Online) [Internet]. 2015 [citado em 5 mar 2017]; 23(3):543-52. Disponível em: http://www.redalyc.org/ articulo.oa?id=281442223024 2

24. Santos CLM, Rodrigues CLP, Silva LB, Bakke HA, Leite ASM, Leal MMA. Fatores de estresse na atividade de médicos em João Pessoa (PB, Brasil). Produção [Internet]. 2011 [citado em 06 set 2017]; 21(1):181-9. Disponível em: http://www.scielo.br/pdf/prod/v21n1/ AOP_200811118.pdf

25. Anjos DR, Silva EA, Falqueiro HJA, Freitas PMP, Peres VPM Massruhá VC, et al. Estresse: fatores desencadeantes, identificação e avaliação de sinas e sintomas no enfermeiro atuante em UTI neonatal. Rev. Inst. Ciênc. Saúde [Internet]. 2008 [citado em 20 fev 2017]; 26(4):426-31. Disponível em: http://files.bvs.br/ upload/S/0104-1894/2008/v26n4/a1741.pdf.

26. Klein SD, Bucher HU, Hendriks MJ, Baumann-Hölzle R, Streuli JC, Berger TM et al. Sources of distress for physicians and nurses working in Swiss neonatal intensive care units. Swiss Med. Wkly [Internet]. 2017 [cited 2017 Sep 21]; 147:1447. Available from: https://www.ncbi.nlm.nih.gov/pubmed/28804867

27. McCarthy VJC, Power S, Greiner BA. Perceived occupational stress in nurses working in Ireland. Occup Med [Internet]. 2010 [cited 2017 Oct 13]; 60(8):604-10. Availablefrom: http://occmed. oxfordjournals.org/content/60/8/604.full.pdf+html.

28. Panunto MR, Guirardello EB. Ambiente da prática profissional e exaustão emocional entre enfermeiros de terapia intensiva. Rev. latinoam. enferm. (Online) [Internet]. 2013 [citado em 3 mar 2017]; 21(3):1-8. Disponível em: http://www.scielo.br/pdf/rlae/v21n3/ pt 0104-1169-rlae-21-03-0765.pdf

29. Medeiros SM, Ribeiro LM, Fernandes SMBA, Veras VSD. Condições de trabalho e enfermagem: a transversalidade do sofrimento no cotidiano. Rev. eletrônica enferm. [Internet]. 2006 [citado em 6 mar 2017]; 8(2):233-40. Disponível em: http://www.fen.ufg.br/ revista/revista8_2/pdf/v8n2a08.pdf

30. Santos JLG, Silva RM, Prochnow AG, Leite JL, Silva DC, Erdmann AL. Prazer e sofrimento no exercício gerencial do enfermeiro no contexto hospitalar. Esc. Anna Nery Rev. Enferm [Internet]. 2013 [citado em 6 mar 2017]; 17(1):97-103. Disponível em: http://www. scielo.br/pdf/ean/v17n1/14.pdf 